\title{
The cathepsin S cysteine proteinase of the burrowing nematode Radopholus similis is essential for the reproduction and invasion
}

Ke Wang ${ }^{1 \dagger}, \mathrm{Yu} \mathrm{Li}^{1,2+}, \mathrm{Xin} \mathrm{Huang}^{1}$, Dong-wei Wang ${ }^{1}$, Chun-ling $\mathrm{Xu}^{1}$ and Hui Xie ${ }^{1^{*}}$

\begin{abstract}
Background: The nematode Radopholus similis is an important migratory endoparasite of plants. Cysteine proteinases such as cathepsin S (CPS) play key roles during embryonic development, invasion, and pathogenesis in nematodes and many other animal parasites. This study was designed to investigate the molecular characterization and functions of a cathepsin S protease in $R$. similis and to find new targets for its control.

Results: $\mathrm{Rs}$-CPS of $\mathrm{R}$. similis, $\mathrm{Hg}$-CPS of Heterodera glycines and Ha-CPS of $\mathrm{H}$. avenae are closely genetically related and share the same branch of the phylogenetic tree. Rs-cps is a multi-copy gene that is expressed in the esophageal glands, ovaries, testes, vas deferens, and eggs of $R$. similis. Rs-cps mRNA transcripts are expressed at varying levels during all developmental stages of $R$. similis. Rs-cps expression was highest in females. The neurostimulant octopamine did not significantly enhance the ingestion of the dsRNA soaking solution by $R$. similis but instead had a detrimental effect on nematode activity. The dsRNA soaking solution diffused into the body of $R$. similis not only through the esophageal lumen but also through the amphids, excretory duct, vagina, anus and cloacal orifice. We confirmed that RNAi significantly suppressed the expression level of Rs-cps and reproductive capability and pathogenicity of $R$. similis.
\end{abstract}

Conclusions: Our results demonstrate that Rs-cps plays important roles in the reproduction, parasitism and pathogenesis of $R$. similis and could be used as a new potential target for controlling plant parasitic nematodes.

Keywords: Radopholus similis, Cysteine proteinase, Cathepsin S, RNAi, Reproduction, Pathogenicity

\section{Background}

The burrowing nematode Radopholus similis is a migratory endoparasite of plants. $R$. similis is one of the most destructive plant pathogenic nematodes in the world and is listed as a quarantine pest in many countries and regions $[1,2] . R$. similis has a wide host range and attacks more than 250 plant species [3]. R. similis severely harms banana, citrus, pepper, coffee and other agronomic and horticultural crops $[4,5]$ and is the most serious plant pathogenic nematode in most banana-growing areas of the world [6]. Despite extensive attention and research,

\footnotetext{
*Correspondence: xiehui@scau.edu.cn

${ }^{\dagger}$ Ke Wang and Yu Li contributed equally to this work

${ }^{1}$ Laboratory of Plant Nematology and Research Center of Nematodes

of Plant Quarantine, Department of Plant Pathology, South China

Agricultural University, Guangzhou 510642, China

Full list of author information is available at the end of the article
}

controlling $R$. similis remains problematic worldwide, and effective approaches must be explored and established.

Proteolytic enzymes can be classified into four main groups: cysteine, serine, aspartyl and metallo proteinases. Cysteine proteinases are the most extensively studied [7]. Cysteine proteinases (EC 3.4.22) have been identified in a variety of organisms [8]. Most cysteine proteases are expressed and proteolytically active in the intestines, and these enzymes are the main digestive enzymes in nematodes and animal parasites [7]. Cysteine proteinases play important roles in embryogenesis and development, infection, parasitism, pathogenesis and immune evasion in nematodes and many other animal parasites [9-12]. Nematode cysteine proteinases mainly include cathepsin B-, L-, S-, K- and Z-like cysteine proteinases, and cathepsin L (CL) and cathepsin B (CB) have been extensively 
studied in recent years. CL is essential for embryogenesis and development in Caenorhabditis elegans [10, 13]. Guiliano et al. [14] demonstrated that CL proteinases in filarial nematodes are associated with larval molting and cuticle and eggshell remodeling. CB plays important roles in molting, the successful development of Onchocerca volvulus fourth-stage larvae [15] and in the invasion and pathogenesis of Fasciola hepatica and Angiostrongylus cantonensis [16, 17]. At present, CB genes have rarely been cloned in plant parasitic nematodes, and only CB of Bursaphelenchus xylophilus (GenBank No: GU130153) and R. similis (GU360972) are cloned. However, many CL genes have been cloned in plant parasitic nematodes, such as Heterodera avenae (ACJ13100), $H$. glycines (Y09498), H. schachtii (ACJ13098), Globodera virginiae (ACJ13094), G. Mexicana (ACJ13096), Meloidogyne incognita (CAD89795), Rotylenchulus reniformis (AAY45870) and B. xylophilus (ACH56225) [7, 18, 19]. Li et al. $[19,20]$ reported that $R s-c b-1$ plays key roles in reproduction, development, hatching and pathogenesis in $R$. similis. However, the cathepsin $\mathrm{S}$ gene (cps) has rarely been reported, and only the cps genes of H.glycines [7], R. similis (EU659125) and H. avenae [21] have been cloned. The functions of cps in plant parasitic nematodes have not been explored. In this study, the expression and tissue localization of Rs-cps in $R$. similis were investigated using qPCR and in situ hybridization, and the roles of $R s$ cps during reproduction and pathogenesis were studied using RNAi combinated with inoculation of carrot callus and tomato plants in pots. This study is the first to examine the functions of $c p s$ in plant parasitic nematodes and suggests a promising new target for controlling $R$. similis.

RNAi is a means by which dsRNA (double stranded RNA) induces sequence-specific posttranscriptional gene silencing [22]. This method was first developed in C. elegans and has subsequently been used in organisms ranging from lower fungi to higher mammals [22-26]. RNAi is also a very powerful tool for examining the functions of genes in plant nematodes and other organisms. For plant parasitic nematodes, in vitro RNAi (performed by soaking the nematodes in a solution of dsRNA in vitro) is the most widely used method, but the soaking time required to obtain optimal RNAi efficiency differs greatly among nematode species [19, 27-33]. The feeding mechanisms of plant parasitic nematodes vary. The infective second-stage juveniles of sedentary endoparasitic nematodes (such as cyst and root-knot nematodes) feed only following the establishment of a feeding site inside the root. Therefore, the primary barrier to successful in vitro RNAi is ensuring the ingestion of the dsRNA by the non-feeding second-stage juveniles of plant parasitic nematodes. In second-stage juveniles of G. pallida, H. glycines and M. incognita, dsRNA uptake can be induced by adding the neurotransmitter octopamine or resorcinol to successfully silence the targeted genes [27, 28]. In vitro RNAi-induced gene silencing has been achieved in B. xylophilus without exogenous neurotransmitter [31, 32]. The in vitro dsRNA soaking method has been used to induce RNAi to $R$. similis $[19,20,34]$, but whether neurotransmitters facilitate the uptake of dsRNA by $R$. similis and affect nematode activity have not been examined. This study examined these questions using FITC as a visual marker.

\section{Methods}

\section{Ethics statement}

Animals were treated in strict accordance with the Animal Ethics Procedures and Guidelines of the People's Republic of China. All animal procedures were approved by the Animal Ethics Committee of the South China Agricultural University.

\section{Nematode inoculum and plant growth conditions}

Radopholus similis was collected from the roots of the ornamental plant Anthurium andraeanum and cultured in vitro on carrot disks at $25^{\circ} \mathrm{C}$ [35]. At $50 \mathrm{~d}$ after inoculation, the cultured nematodes were extracted from the carrot disks according to the method described by Zhang et al. [34]. The tomato seeds used in this study were purchased from Guangzhou Changhe Seed Limited Company, Guangdong, and surface sterilized as described by Arshad et al. [36]. The sterilized seeds were sown in $1.5 \mathrm{~L}$ of sterilized soil and cultured in a $25{ }^{\circ} \mathrm{C}$ growth cabinet (16 h light/8 h dark photoperiods) for $30 \mathrm{~d}$ [20].

\section{RNA extraction, PCR amplification of Rs-cps and phylogenetic analysis}

Total RNA was extracted from 20,000 mixed-stages nematodes of $R$. similis using TRIzol reagent (Invitrogen) and verified as previously described [34]. After being treated with RQ1 RNase-Free DNase (Promega) for $15 \mathrm{~min}$ at $37{ }^{\circ} \mathrm{C}$, the cDNA was synthesized using the Reverse Transcriptase M-MLV (Takara). The full-length cDNA sequence of Rs-cps was amplified using the specific primers Rs-cps-S1 and Rs-cps-A1 (Table 1). The PCR product was purified, ligated into the vector pMD20-T (Takara) and transformed into Escherichia coli DH5a competent cells. Positive clones were confirmed by sequencing and the recombinant plasmid pMD20-Rs-cps was extracted for later use.

The amino acid sequences of the Rs-CPS protein and other CPS proteins were aligned using ClustalW. Based on the amino acid sequences of 25 CPS proteins from 17 species, a phylogenetic tree was constructed using the neighbor-joining method in MEGA 5.1 [37]. Bootstrap values were calculated from 1000 replicates. 
Table 1 Primers used in this study

\begin{tabular}{|c|c|c|}
\hline Primer name & Sequence & Primer use \\
\hline Rs-cps-S1 & 5'-AGTGCCCCTCCGAAATGT-3' & RT-PCR \\
\hline Rs-cps-A1 & 5'-TGTCCGTTCTTCCGTTCA-3' & \\
\hline SF & 5'-TGTTGGCGGTCCCTGTG-3' & Southern blot \\
\hline SR & 5'-CGTGTTCGTGGACGGAGTT-3' & \\
\hline Ish-T7S1 ${ }^{\mathrm{a}}$ & 5'-GGATCCTAATACGACTCACTATAGGGTGTTGGCGGTCCCTGTG-3' & ISH template \\
\hline $\mid s h-A 1$ & 5'-CCGTTCTCCTCGATGTAGTCA -3' & \\
\hline Ish-S2 & 5'-TGTTGGCGGTCCCTGTG-3' & ISH template \\
\hline Ish-T7A2 ${ }^{\mathrm{a}}$ & 5'-GGATCCTAATACGACTCACTATAGGGCCGTTCTCCTCGATGTAGTCA-3' & \\
\hline qPCR-F & 5'-AGAACTCCGTCCACGAACAC-3' & qPCR \\
\hline qPCR-A & 5'-GCCCACATTGCGCTTGCT-3' & \\
\hline Actin- $\mathrm{F}$ & 5'-GAAAGAGGGCCGGAAGAG-3' & qPCR \\
\hline Actin-R & 5'-AGATCGTCCGCGACATAAAG-3' & \\
\hline CPS-T7S ${ }^{a}$ & 5'-GGATCCTAATACGACTCACTATAGGGTGTTGGCGGTCCCTGTG-3' & dsRNA template \\
\hline CPS-A & 5'-CGTGTTCGTGGACGGAGTT-3' & \\
\hline CPS-S & 5'-TGTTGGCGGTCCCTGTG-3' & dsRNA template \\
\hline CPS-T7A $A^{a}$ & 5'-GGATCCTAATACGACTCACTATAGGG CGTGTTCGTGGACGGAGTT-3' & \\
\hline eGFP-T7S ${ }^{a}$ & 5'-GGATCCTAATACGACTCACTATAGGGCAGTGCTTCAGCCGCTACC-3' & dsRNA template \\
\hline eGFP-A & 5'-AGTTCACCTTGATGCCGTTCTT-3' & \\
\hline eGFP-S & 5'-CAGTGCTTCAGCCGCTACC-3' & dsRNA template \\
\hline eGFP-T7 $A^{a}$ & 5'-GGATCCTAATACGACTCACTATAGGGAGTTCACCTTGATGCCGTTCTT-3' & \\
\hline
\end{tabular}

a The T7 promoter sequence is italiced

\section{Southern blot hybridization}

Approximately $10 \mu \mathrm{g}$ of gDNA was obtained from R. simi$l i s$ and digested with NdeI and EcoRI. The digested DNA products were separated by $0.8 \%(\mathrm{w} / \mathrm{v})$ agarose gel electrophoresis and transferred to a Hybond $\mathrm{N}+$ membrane (Amersham) [38]. A 438-bp DIG-labeled probe was prepared using a PCR DIG Probe Synthesis Kit (Roche) with the specific primers SF and SR (Table 1). The membrane was hybridized for $18 \mathrm{~h}$ at $54.5^{\circ} \mathrm{C}$ with the probe. Hybridization was performed using a Dig High Primer DNA Labeling and Detection Starter Kit I (Roche) according to the manufacturer's instructions. After hybridization, the membrane was washed with $2 \times$ SSC/0.1\% SDS for $15 \mathrm{~min}$ at $25{ }^{\circ} \mathrm{C}$ followed by $0.5 \times \mathrm{SSC} / 0.1 \% \mathrm{SDS}$ for $30 \mathrm{~min}$ at $65^{\circ} \mathrm{C}$ and examined. An equal amount of carrot callus gDNA was used as a control.

\section{In situ hybridization}

In situ hybridization was performed as described by De Boer et al. [39] and Cheng et al. [40]. Specific sense (IshT7S1, Ish-A1) and antisense (Ish-S2, Ish-T7A2) primers (Table 1) were designed to amplify a 478-bp fragment based on the full-length sequence of Rs-cps. The purified PCR product served as the template to synthesize DIGlabeled sense and antisense RNA probes using DIG RNA labeling mix (Roche) according to the manufacturer's instructions. Following fixation, the intact nematodes were cut into 2-5 fragments and hybridized with the DIG-labeled RNA probes (300 ng/mL). After hybridization, the stained nematode sections were examined and photographed using a 90i differential interference microscope (Nikon).

\section{Expression analysis of $R s-c p s$ and qPCR}

qPCR was used to detect the expression levels of $R s$ cps in $R$. similis at different developmental stages. Total RNA samples were extracted from $100 R$. similis females, males, eggs and juveniles using an RNeasy Micro kit (Qiagen), respectively. The extracted RNA was treated and quantified as previously described [34]. cDNA was synthesized using an iScript cDNA synthesis kit (Bio-Rad) according to the manufacturer's instructions. Specific primers, qPCR-A and qPCR-S (Table 1), were designed according to the full-length sequence of $R s$-cps to detect $R s$-cps expression levels in $R$. similis. $\beta$-actin was amplified as a reference gene using the primers Actin-F/Actin-R (Table 1) [41]. qPCR was performed on a CFX-96 qPCR machine using iTaq Universal SYBR Green Supermix (Bio-Rad). The initial data analysis was performed using CFX-96 manager software, which created $\mathrm{Ct}$ values and extrapolated the relative levels of PCR products from standard curves. Melt curves were obtained routinely, which allowed the possibility of both contamination and primer dimers to be discounted [34, 
$38,40]$. All experiments were performed in triplicate with three biological replicates [20].

\section{Synthesis of Rs-cps dsRNA of $R$. similis}

The specific primers CPS-T7S/CPS-A and CPS-S/CPST7A (Table 1 ) were designed to amplify a 438-bp fragment containing the T7 promoter. The purified PCR product was used to transcribe $R s$-cps sense and antisense single-stranded RNA (ssRNA) using a ScriptMAXTM Thermo T7 Transcription Kit (TOYOBO). The corresponding dsRNA was synthesized and purified as described by Hannon [42]. The non-endogenous control dsRNA (the enhanced green fluorescent protein gene, e $g f p$ ) was synthesized using the specific primers eGFPT7S/eGFP-A and eGFP-S/eGFP-T7 (Table 1).

\section{Effects of soaking R. similis with FITC and octopamine}

The effects of FITC and octopamine on nematode activity and the effect of octopamine on dsRNA solution uptake by $R$. similis were assessed using FITC as a visual marker. The RNAi soaking method was performed as previously described [27]. Approximately 20,000 mixed-stage nematodes were collected from carrot disks and soaked in M9 buffer. The following compounds respectively were added to the above soaking solution at the indicated concentrations: (I) 0 (CK), $0.1,0.2,0.4,0.8,1.0$ or $2.0 \mathrm{mg} / \mathrm{mL}$ fluorescein isothiocyanate (FITC) (Sigma-Aldrich) (stock made up at $20 \mathrm{mg} / \mathrm{mL}$ in DMF) [27]; (II) 0 (CK), 10, $25,50,75,100$ or $200 \mathrm{mM}$ neurostimulant octopamine (Sigma-Aldrich); (III) FITC $(0.8 \mathrm{mg} / \mathrm{mL})$ or FITC $(0.8 \mathrm{mg} /$ $\mathrm{mL})$ plus octopamine $(50 \mathrm{mM})$. Rs-cb-1 dsRNA was added to the soaking solution at a final concentration of $2.0 \mathrm{mg} / \mathrm{mL}$ [27]. Nematodes not treated with FITC or octopamine were used as controls. The nematodes were maintained in $1 \mathrm{~mL}$ of soaking solution with gentle agitation $(100 \mathrm{rpm})$ in a dark rotary incubator at $25^{\circ} \mathrm{C}$ for 4,8 , 12 and $24 \mathrm{~h}$. After incubation, the nematodes were transferred to a $10-\mathrm{mL}$ centrifuge tube and washed six times with sterile water to remove the soaking solution. FITC uptake was measured based on fluorescence intensity, and the effects of FITC and octopamine on nematode activity were detected using a fluorescence microscope (Nikon 90i) with appropriate filters. The nematodes were considered dead if they did not move after being pricked with a platinum wire [32]. For each treatment, 100 nematodes were effectively counted to quantify the effects of soaking $R$. similis with FITC and octopamine. Five biological replicates were performed.

\section{Effects of Rs-cps silencing on $R$. similis}

Approximately 1000 mixed-stage nematodes were washed with DEPC water and then soaked in Rs-cps dsRNA solution $(2.0 \mathrm{mg} / \mathrm{ml})$ with gentle agitation in a dark rotary incubator $\left(100 \mathrm{rpm}, 25^{\circ} \mathrm{C}\right)$ for $12,24,36$ and $48 \mathrm{~h}$, respectively. The treated nematodes were used in the following experiments. (I) Total RNA was extracted from 100 nematodes in each treatment group after they were washed with DEPC water, and qPCR was used to detect the silencing efficiency of Rs-cps in $R$. similis, as described above. These experiments were performed in triplicate with three biological replicates. (II) The phenotypic changes of nematodes were observed after soaking in Rs-cps dsRNA solution for $36 \mathrm{~h}$. (III) A total of 30 female nematodes were inoculated onto carrot disks and cultured for $50 \mathrm{~d}$ at $25^{\circ} \mathrm{C}$, and then the reproductive rates (reproductive rate $=$ final nematodes $/$ initial nematodes) of the nematodes were calculated. Non-endogenous e $g f p$ dsRNA treated nematodes $(2.0 \mathrm{mg} / \mathrm{mL})$ were used as a control. The soaking times for the controls were the same as those used for the Rs-cps dsRNAs. Untreated nematodes were used as a blank control (CK).

To detect the effect of Rs-cps silencing on the pathogenicity of $R$. similis, 1000 mixed-stage nematodes treated with $R s$-cps dsRNA $(2.0 \mathrm{mg} / \mathrm{mL})$ for $36 \mathrm{~h}$ were inoculated onto each of the selected tomato plantlets. The selected plantlets were identical in height (approximately $20 \mathrm{~cm}$ ) and growth conditions and were cultivated in a greenhouse as described above [20]. Nematodes treated with e $g f p$ dsRNA for $36 \mathrm{~h}$ were used as the control. Untreated nematodes were used as a blank control. The plantlets were managed as usual except that they were not watered for the first 5 days [34]. After 60 days, the plant heights, fresh shoot weights and fresh root weights of the plants were measured and recorded. The symptoms of infected roots were photographed. The nematodes in the rhizosphere were isolated and quantified as described elsewhere [34, 43]. Five biological replicates were performed.

\section{Statistical analysis}

All data in this study were analyzed using SAS 9.2 (SAS Institute, Cary, NC, USA) and subjected to one-way analysis of variance (ANOVA), and differences between treatments were compared using Duncan's Multiple Range Test at $p=0.05$.

\section{Results}

\section{PCR amplification of Rs-cps and phylogenetic analysis}

The 974-bp full-length cDNA sequence of Rs-cps was amplified using the specific primers Rs-cps-S1 and Rscps-A1 (Table 1) and confirmed by sequencing (result not shown). The sequencing results were consistent with the sequence in GenBank (GenBank: EU659125). The recombinant pMD20-Rs-cps plasmid, which includes the intact ORF (945 bp) of Rs-cps, was extracted for later use. The Rs-CPS protein encodes 314 aa with a theoretical molecular mass of $34.7 \mathrm{kDa}$. A 17 aa signal peptide with 
a cleavage site between Ala17 and Val18 was predicted by SignalP 3.0 at the N-terminus of the deduced Rs-CPS sequence. No transmembrane helix was predicted from the deduced Rs-CPS sequence using the TMHMM 2.0. The amino acid sequences alignment results revealed 56 and $45 \%$ identity of Rs-CPS (ACH56227) with $\mathrm{Hg}$ CPS from $H$. glycines (CAA70694) and $\mathrm{Ha}$-CPS from $\mathrm{H}$. avenae (AGL80530), respectively. A phylogenetic tree was generated using the neighbor-joining method based on the amino acid sequences of CPS proteins (Fig. 1). Twenty-five CPS proteins from 17 species were divided into three groups. Rs-CPS of $R$. similis, $H g$-CPS of $H$. glycines and $\mathrm{Ha}$-CPS of $\mathrm{H}$. avenae were present in the same branch of the phylogenetic tree, within the Nematoda

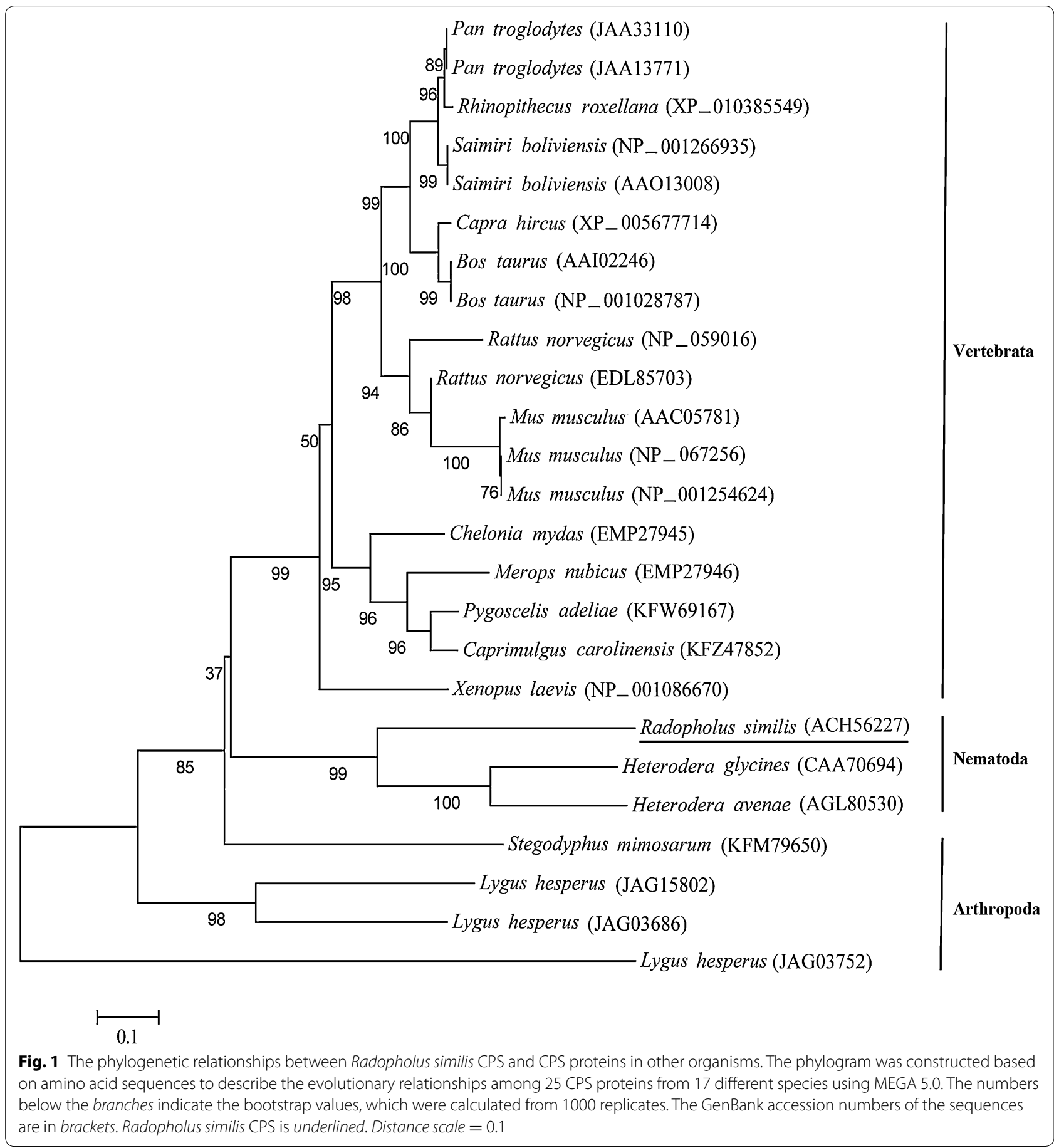


group, suggesting a close phylogenetic relationship. The remaining 22 CPS proteins were divided into two groups: Vertebrata and Arthropoda (Fig. 1).

\section{Southern blot hybridization}

To determine the gene copy number of Rs-cps in R. similis, $R$. similis gDNA was analyzed by Southern blot. The gDNA samples were digested with NdeI and EcoRI and then hybridized with a 438-bp DIG-labelled probe. Four strong hybridizing bands were observed in the NdeI- and EcoRI-digested $R$. similis gDNA samples. No hybridization signal was detected for digested carrot callus gDNA. Because there are no NdeI or EcoRI restriction sites in the genomic coding region or the cDNA sequence of Rs-cps, the results suggested that Rs-cps exists as a multi-copy gene in the R. similis genome (Fig. 2).

\section{Tissue localization and expression of Rs-cps mRNA in $R$. similis}

The tissue localization of Rs-cps in R. similis was determined by in situ hybridization. Rs-cps mRNA was expressed in the esophageal glands and ovaries of females (Fig. 3a, b, d), the esophageal glands, testes and vas deferens of males (Fig. 3f, h), and the eggs of $R$. similis (Fig. 3j), as indicated by hybridization with a 478-bp DIG-labeled antisense mRNA probe. No hybridization signal was observed in nematodes and eggs when the control sense mRNA probe was used (Fig. 3c, e, g, i, k). The expression level of Rs-cps in $R$. similis at different development stages was detected using qPCR. The results showed that the Rs-cps mRNA transcript was present in all

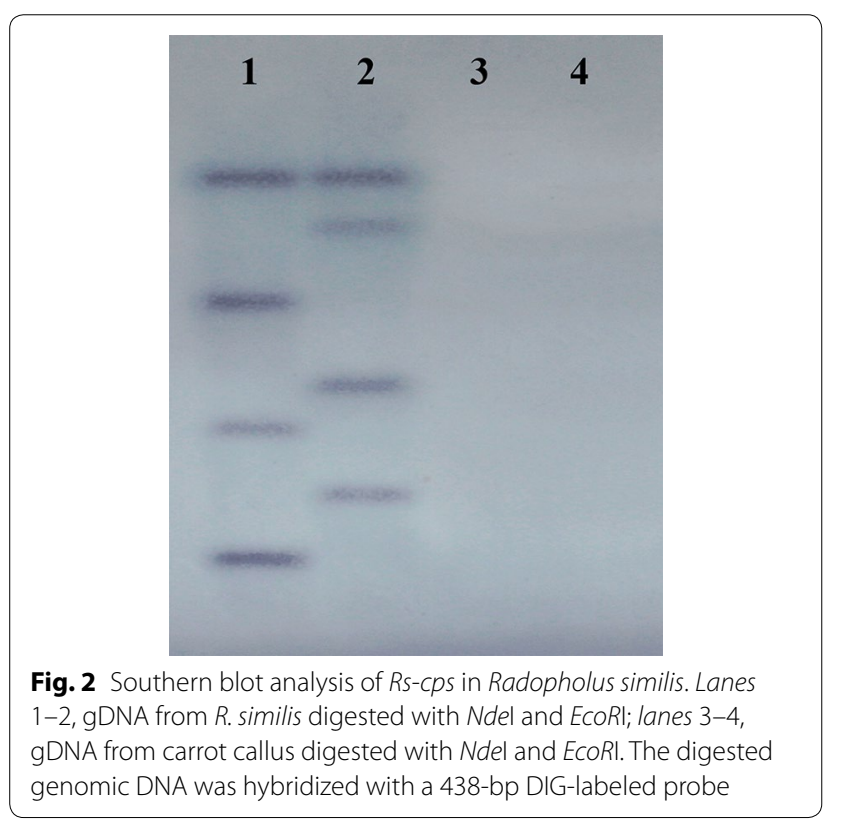

developmental stages of $R$. similis and that the highest transcript level was detected in females. The expression of Rs-cps in juveniles, males and eggs accounted for 64.4, 57.7 and $42.5 \%$ of the expression level in females, respectively. Rs-cps expression was significantly lower in eggs than in juveniles and males $(p<0.05)$, but no significant difference was observed between juveniles and males $(p>0.05)$ (Fig. 3l).

\section{Effects of FITC and octopamine on R. similis}

The effects of FITC and octopamine on the activity of $R$. similis and the effect of octopamine on the uptake of dsRNA solution by nematodes were assessed using FITC as a visual marker. With the exception of the control group (without FITC), the nematodes in the soaking solutions with different concentrations of FITC all fluoresced after $8 \mathrm{~h}$ of incubation, and the fluorescence intensity in the nematodes increased as the concentration of FITC increased. Weak fluorescence was observed in nematodes soaked in solutions containing $0.1,0.2$ and $0.4 \mathrm{mg} /$ $\mathrm{mL}$ FITC, whereas strong fluorescence was observed in nematodes soaked in solutions containing 0.8, 1.0 and $2.0 \mathrm{mg} / \mathrm{mL}$ FITC (Fig. 4a-f). In soaking solutions containing 1.0 and $2.0 \mathrm{mg} / \mathrm{mL}$ FITC, the percentage of inactive nematodes reached 66 and $83.2 \%$, respectively, significantly higher $(p<0.05)$ than the percentage in the control group (without FITC) and the groups incubated in the other four concentrations of FITC $(0.1,0.2,0.4$, $0.8 \mathrm{mg} / \mathrm{mL}$ ). In soaking solutions containing $0.8 \mathrm{mg} / \mathrm{mL}$ FITC, the percentage of inactive nematodes was $15.8 \%$, significantly higher than the percentage in the solution containing $0.1 \mathrm{mg} / \mathrm{mL}$ FITC $(p<0.05)$. However, there was no significant difference $(p>0.05)$ in the percentage of inactive nematodes after the worms were incubated in solutions containing $0.1,0.2$ and $0.4 \mathrm{mg} / \mathrm{mL}$ FITC for $8 \mathrm{~h}$ (Fig. $4 \mathrm{~g}$ ). These results indicate that the FITC was toxic to $R$. similis. The toxic effect of FITC on nematodes was greater at 1.0 and $2.0 \mathrm{mg} / \mathrm{mL}$ than at the other four tested concentrations. There was no significant difference in the activity of $R$. similis when the nematodes were soaked in $0.2,0.4$ and $0.8 \mathrm{mg} / \mathrm{mL}$ FITC $(p>0.05)$. However, more nematodes displayed fluorescence at $0.8 \mathrm{mg} / \mathrm{mL}$ than at 0.2 and $0.4 \mathrm{mg} / \mathrm{mL}$, and the fluorescent intensity was stronger. Therefore, $0.8 \mathrm{mg} / \mathrm{mL}$ FITC was used as a visual marker in the subsequent experiments.

After $8 \mathrm{~h}$ of incubation, there was no significant difference $(p>0.05)$ in the activity of $R$. similis in the groups soaked with octopamine at concentrations of $50 \mathrm{mM}$ or less and the control group (without octopamine). Significant differences were observed in the activity of $R$. similis soaked with octopamine at concentrations greater than $50 \mathrm{mM}$ and the control group $(p<0.05)$. However, activity of nematodes was reduced by only $10.2 \%$ in the group 

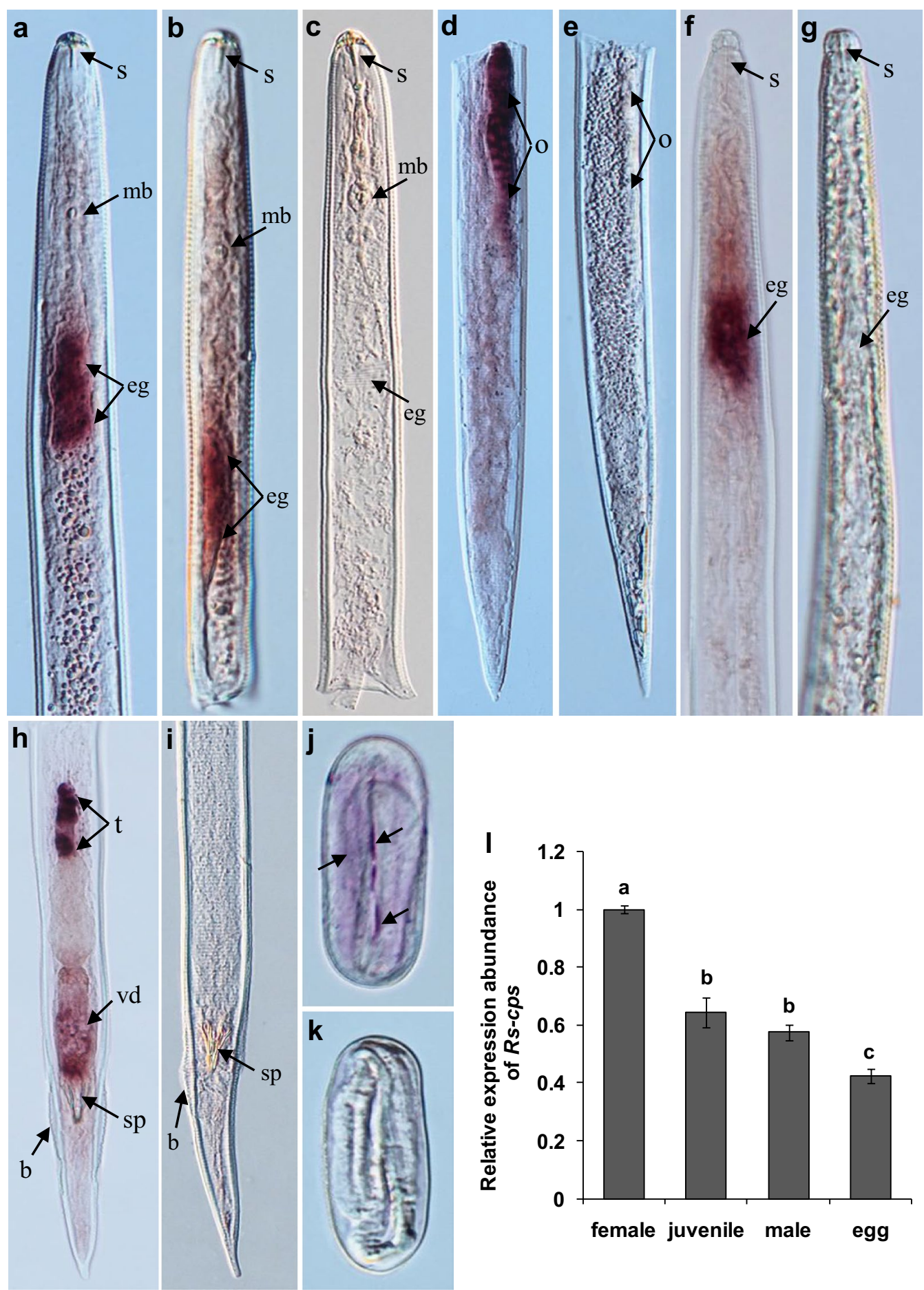

Fig. 3 Tissue localization and expression of Rs-cps in Radopholus similis. a-k Tissue localization of Rs-cps mRNA in R. similis using in situ hybridization. Rs-cps was expressed in the esophageal glands $(\mathbf{a}, \mathbf{b})$ and ovaries $(\mathbf{d})$ of females. Rs-cps was expressed in the esophageal glands (f), testes and vas deferens (h) of males. Rs-cps was expressed in eggs (j). $\mathbf{c}, \mathbf{e}, \mathbf{g}, \mathbf{i}, \mathbf{k}$ no hybridization signal was observed in the control nematodes and eggs that were hybridized with a DIG-labeled sense Rs-cps RNA probe. $b$ bursa; e.g. esophageal glands; $m b$ medium bulb; 0 ovary; $s$ stylet; $s p$ spicules; $v d$ vas deferens; $t$ testis. I Expression of Rs-cps at different development stages in R. similis. The bars indicate the standard errors of the mean $(n=3)$, and different letters indicate significant differences $(p<0.05)$ between treatments 

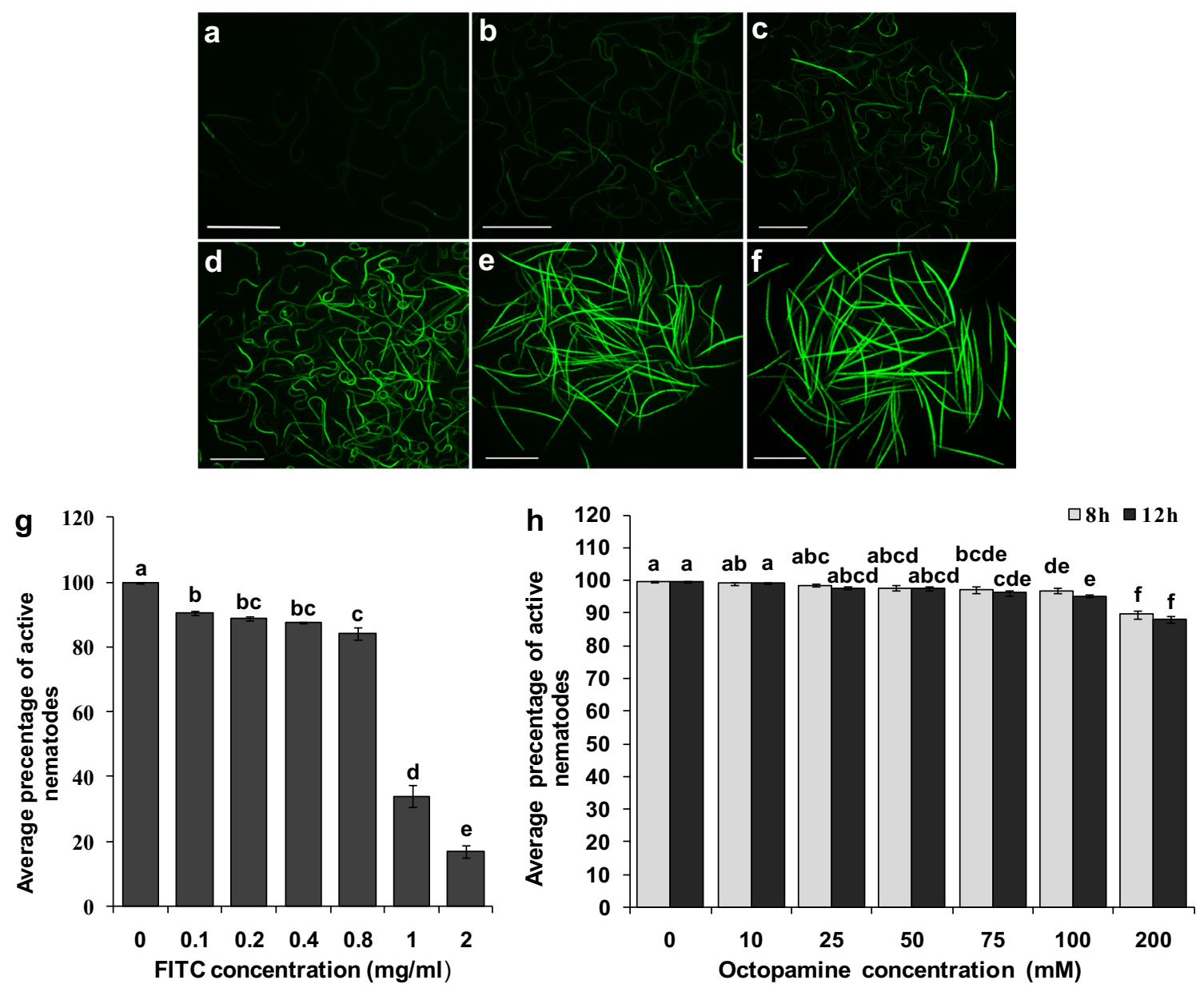

Fig. 4 Effects of soaking mixed stages of Radopholus similis with FITC and octopamine. a-f FITC fluorescence in $R$. similis incubated for $8 \mathrm{~h}$ with different concentrations of FITC, as follows: a $0.1 \mathrm{mg} / \mathrm{mL}$, b $0.2 \mathrm{mg} / \mathrm{mL}, \mathbf{c} 0.4 \mathrm{mg} / \mathrm{mL}$, d $0.8 \mathrm{mg} / \mathrm{mL}$, e $1.0 \mathrm{mg} / \mathrm{mL}$ and f $2.0 \mathrm{mg} / \mathrm{mL}$. The scale bar represents $500 \mu \mathrm{m}$. $\mathbf{g}$ Average percentages of active nematodes after worms were soaked with different concentrations of FITC for $8 \mathrm{~h}$. $\mathbf{h}$ Average percentages of active nematodes after worms were soaked with different concentrations of octopamine for 8 and $12 \mathrm{~h}$. The bars indicate the standard errors of the mean $(n=5)$, and different letters indicate significant differences $(p<0.05)$ between different treatments

soaked with $200 \mathrm{mM}$ octopamine compared to the control group. When the incubation time was extended to $12 \mathrm{~h}$, the activity of $R$. similis soaked in different concentrations of octopamine did not differ from that observed after incubation for $8 \mathrm{~h}$ (Fig. 4h). These results indicate that the detrimental effects of octopamine on the activity of $R$. similis are very small. Therefore, the ability of $50 \mathrm{mM}$ octopamine to enhance the ingestion of the soaking solution by $R$. similis was assessed.

When $0.8 \mathrm{mg} / \mathrm{mL}$ FITC was used as a marker, there was no obvious difference in fluorescence intensity between the mixed-stage $R$. similis that were soaked with $50 \mathrm{mM}$ octopamine and those in soaking solution without octopamine after incubation for $4 \mathrm{~h}$ to $24 \mathrm{~h}$ (Fig. $5 \mathrm{a}-\mathrm{d}$ ). After incubation in FITC solution with or without octopamine for $24 \mathrm{~h}$, the percentage of inactive nematodes reached 50.4 and $43.4 \%$, respectively, significantly higher $(p<0.05)$ than that observed in the control group (6.4\%) and the other incubation time groups (Fig. 5f). These results indicate that octopamine did not significantly enhance the ingestion of soaking solution by $R$. similis but instead had a detrimental effect on nematode activity. Therefore, we directly soaked $R$. similis in dsRNA soaking solution without octopamine in the subsequent RNAi experiments.

After incubation for $8 \mathrm{~h}$ in dsRNA solution with FITC $(0.8 \mathrm{mg} / \mathrm{mL}$ as a visible marker), strong fluorescence signals were observed in the stylet, esophageal lumen, intestine, amphids, excretory duct and anus of $R$. similis (Fig. 6c-e, h). Fluorescence microscopy also indicated that the soaking solution diffused into the body of $R$. similis via the vagina and cloacal orifice (Fig. $6 \mathrm{~g}$, i) and 

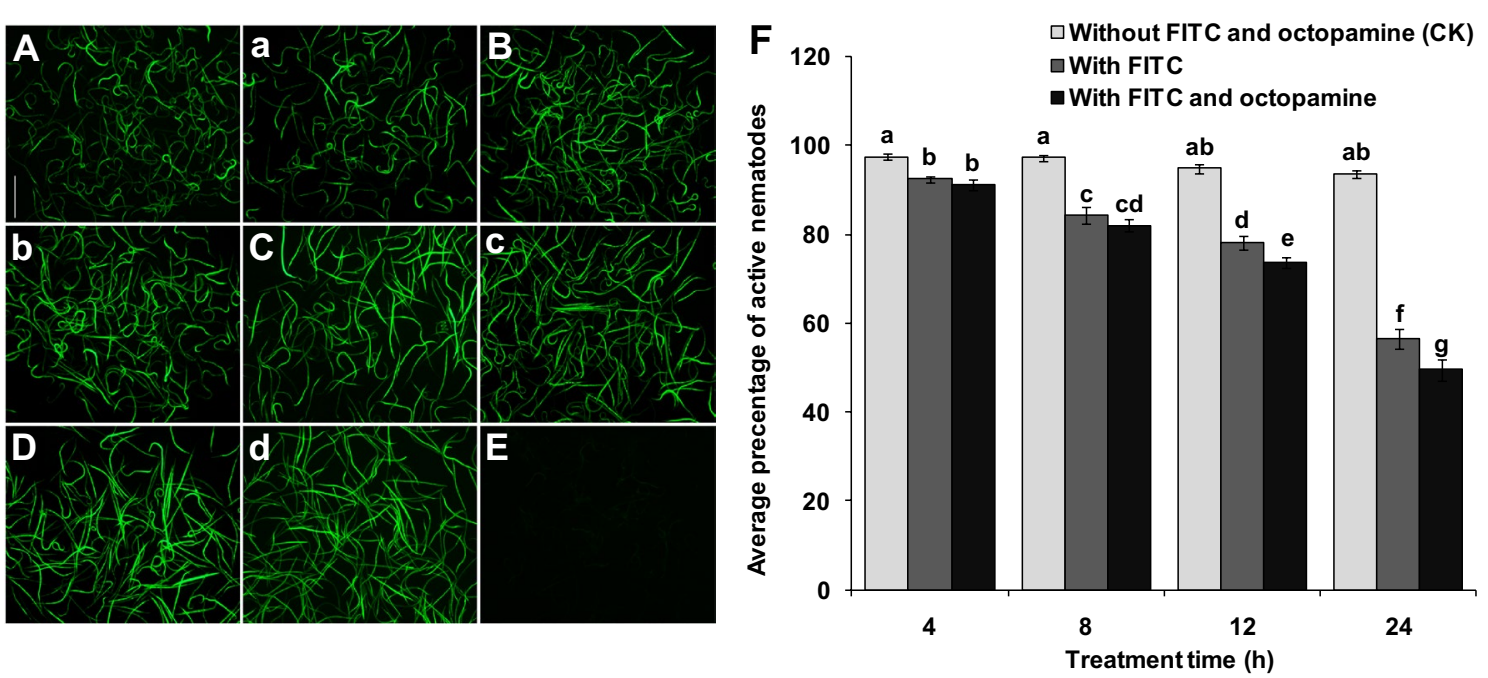

Fig. 5 FITC fluorescence and viability in mixed-stages Radopholus similis incubated for different times in soaking solutions containing FITC and octopamine. a-d Expression in nematodes soaked in $0.8 \mathrm{mg} / \mathrm{mL}$ FITC for 4, 8, 12 and $24 \mathrm{~h}$, respectively. a-d Expression in nematodes soaked in $0.8 \mathrm{~mL} / \mathrm{mg}$ FITC and $50 \mathrm{mM}$ octopamine for 4, 8, 12 and $24 \mathrm{~h}$, respectively. e Nematodes soaked in water without FITC and octopamine were used as a control (CK). The scale bar represents $500 \mu \mathrm{m}$. $\mathbf{f}$ Viability of nematodes after soaking in FITC $(0.8 \mathrm{mg} / \mathrm{mL})$ and octopamine $(50 \mathrm{mM})$ solution for $4,8,12$ and $24 \mathrm{~h}$. The bars indicate the standard errors of the mean $(n=5)$, and different letters indicate significant differences $(p<0.05)$ between treatments

even the egg via the egg shell (Fig. 6a, b). Strong fluorescence signals were observed in these structures. Strong fluorescence signals were observed in the inclusions of the nematodes when the bodies of $R$. similis were pierced using an insect pin (Fig. 6f). Using FITC as a marker, this study is the first to demonstrate that a neurostimulant is not required to induce $R$. similis to ingest dsRNA in a soaking solution via many of its organs in both vermiform nematodes and egg shells.

\section{Detection of RNAi efficiency}

The silencing efficiency of $R s$-cps in $R$. similis was determined by qPCR analysis of worms that were treated with $R s$-cps dsRNA. The results showed that Rs-cps expression levels in nematodes decreased significantly $(p<0.05)$ by $50.5,50.2,74.4$ and $55.4 \%$ when the worms were treated with $R s$-cps dsRNA for $12 \mathrm{~h}, 24 \mathrm{~h}, 36$ and $48 \mathrm{~h}$, respectively, compared to the expression level of Rs-cps in the untreated control nematodes (CK). Rs-cps expression was significantly lower $(p<0.05)$ in the nematodes treated with Rs-cps dsRNA for $36 \mathrm{~h}$ than in the other RNAitreated groups. Rs-cps expression was significantly lower in the nematodes treated with $R s$-cps dsRNA $(p<0.05)$ than in the CK group and the egfp dsRNA-treated nematodes, but there was no significant difference $(p>0.05)$ between the latter two control groups (Fig. 7). Therefore, $R s$-cps expression in $R$. similis was effectively inhibited by soaking the nematodes with Rs-cps dsRNA, and the silencing efficiency was highest at $36 \mathrm{~h}$.

\section{Phenotype and reproduction of $R$. similis after RNAi}

After soaking the nematodes in Rs-cps dsRNA for $36 \mathrm{~h}$, most of the $R$. similis became twisted and circled with trembling (Fig. $8 \mathrm{~b}-\mathrm{c}$ ). The effect of Rs-cps silencing on the reproductive capabilities of $R$. similis was assessed by inoculating the nematodes on carrot disks. After culture on carrot disks for $50 \mathrm{~d}$, nematodes treated with Rs-cps dsRNA for 12, 24, 36 and 48 h had a significantly lower reproductive rate (reproductive rate $=$ final nematodes / initial nematodes) than untreated (CK) and egfp dsRNA-treated nematodes $(p<0.05)$. Nematodes treated with Rs-cps dsRNA for $36 \mathrm{~h}$ had the lowest reproductive rate (65.1), but no significant difference $(p>0.05)$ was observed between the 36 and 48 -h treatments. The reproductive rates of the nematodes treated with $R s$-cps dsRNA for 36 and $48 \mathrm{~h}$ were significantly lower $(p<0.05)$ than those treated for $12 \mathrm{~h}$ (149.3) and $24 \mathrm{~h}$ (240.8), but no significant difference $(p>0.05)$ was observed between the latter two groups. The reproductive rates of the untreated and egfp dsRNA-treated nematodes were higher than 490 , and no significant difference $(p>0.05)$ was observed among the untreated and egfp dsRNAtreated groups (Fig. 8a).

\section{The pathogenicity of $R$. similis decreases significantly after RNAi}

The effect of Rs-cps RNAi on the pathogenicity of $R$. similis was analyzed by inoculating nematodes on tomato plants under different treatment conditions. At $60 \mathrm{~d}$ 

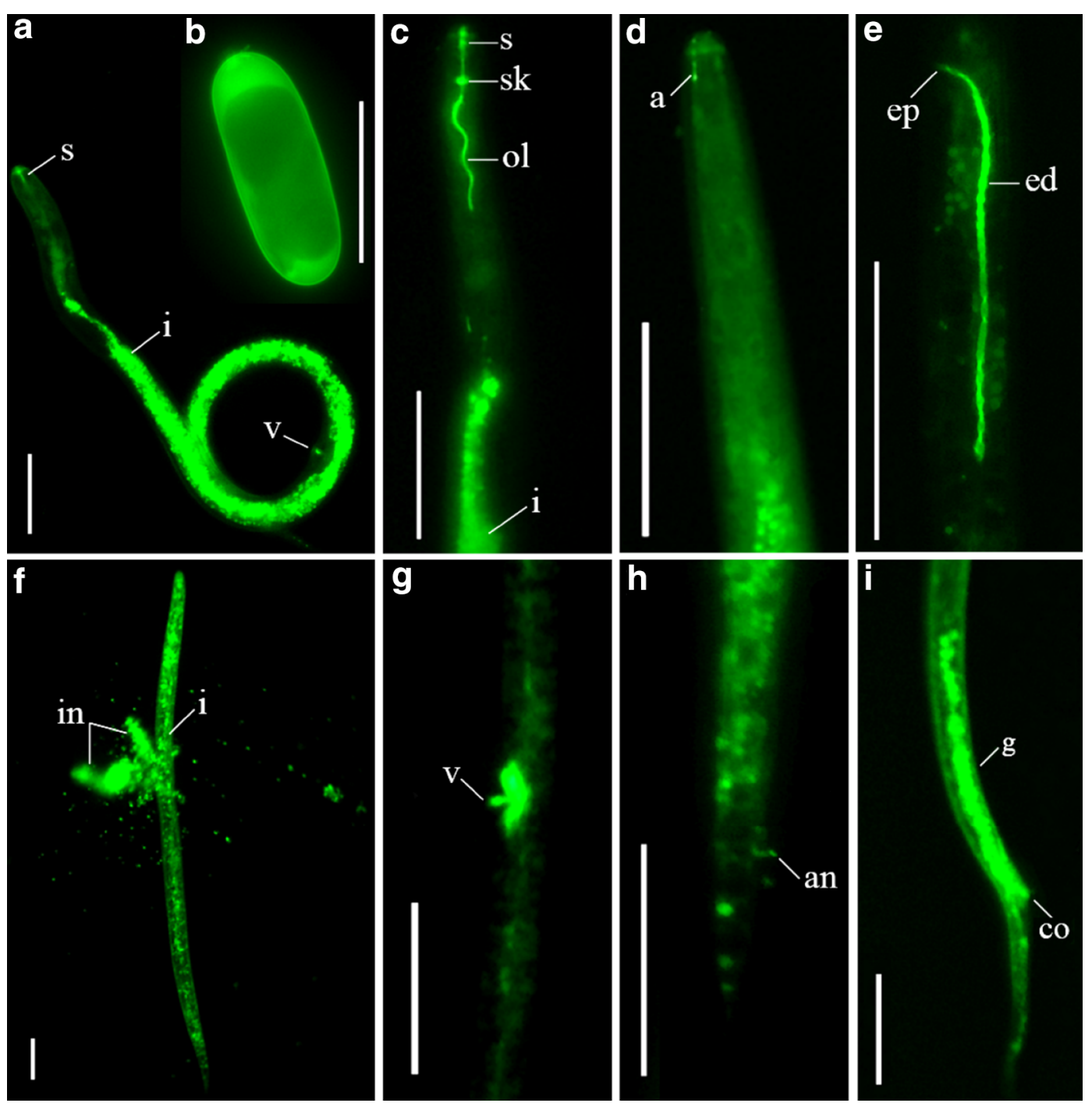

Fig. 6 Fluorescence microscopy showing FITC and dsRNA uptake by Radopholus similis soaked in $0.8 \mathrm{mg} / \mathrm{mL}$ FITC for $8 \mathrm{~h}$. a, b FITC fluorescence in the nematode and egg. $\mathbf{c}$ FITC fluorescence in the style, the esophageal lumen and the intestines in $R$. similis. $\mathbf{d}$ FITC fluorescence in the amphids. $\mathbf{e}$ A strong FITC signal was observed in the excretory ducts of $R$. similis. $\mathbf{f}$ A strong fluorescence signal was observed in the inclusion of the nematode body. $\mathbf{g}$ FITC fluorescence was observed in the vagina. $\mathbf{h}$ FITC entered the rectum via the anus. $\mathbf{i}$ FITC entered the reproductive system via the cloacal orifice. $a$ amphids; an anus; co cloacal orifice; ed excretory duct; ep excretory pore; $g$ gonads; $i$ intestine; in inclusion of the nematode body, ol oesophageal lumen; s stylet; sk stylet knobs; $v$ vagina. The scale bar represents $50 \mu \mathrm{m}$

after inoculation, the plant heights, fresh shoot weights and fresh root weights of the tomato plants inoculated with the nematodes treated with Rs-cps dsRNA for $36 \mathrm{~h}$ were $81.12 \mathrm{~cm}, 82.44$ and $12.81 \mathrm{~g}$, respectively, significantly higher $(p<0.05)$ than the plants inoculated with untreated nematodes $(56.22 \mathrm{~cm}, 55.56$ and $5.27 \mathrm{~g}$, respectively) or e $f f p$ dsRNA-treated nematodes $(58.42 \mathrm{~cm}, 53.02$ and $5.82 \mathrm{~g}$, respectively) but lower than those of uninoculated healthy plants $(89.52 \mathrm{~cm}, 96.74$ and $18.55 \mathrm{~g}$, respectively). There was a significant difference in fresh shoot weights and fresh root weights between uninoculated healthy tomato plants (CK) and the plants inoculated with nematodes treated with $R s-c p s$ dsRNA for $36 \mathrm{~h}(p<0.05)$, but no significant difference in plant height was observed between the two treatments $(p>0.05)$. There was no significant difference $(p>0.05)$ in the three growth parameters between tomato plants inoculated with e $g f p$ dsRNA-treated and untreated nematodes (Fig. 9a-c). The number of nematodes in the rhizospheres of tomato plants inoculated with nematodes that were treated with Rs-cps dsRNA for $36 \mathrm{~h}$ was 742 , significantly lower $(p<0.05)$ than that observed in the 


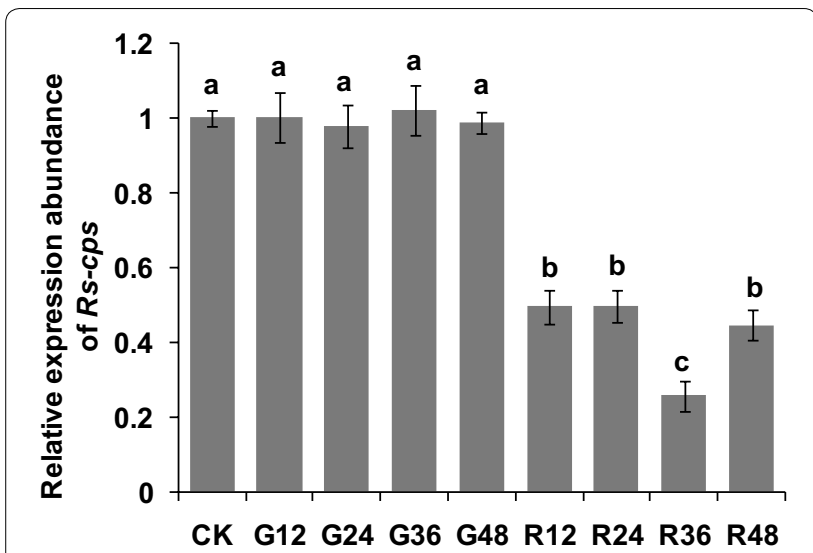

Fig. $\mathbf{7}$ Expression of the Rs-cps mRNA in Radopholus similis treated with Rs-cps dsRNA for different times. CK, untreated nematodes; G12, $\mathrm{G} 24, \mathrm{G} 36$ and $\mathrm{G} 48$, expression in nematodes soaked in non-endogenous egfp dsRNA for 12 h, 24 h, 36 h and 48 h, respectively; R12, R24, R36 and R48, expression in nematodes soaked in Rs-cps dsRNA for 12 h, 24 h, 36 h and 48 h, respectively. The bars indicate the standard errors of the mean $(n=3)$, and different letters indicate significant differences $(p<0.05)$ between treatments

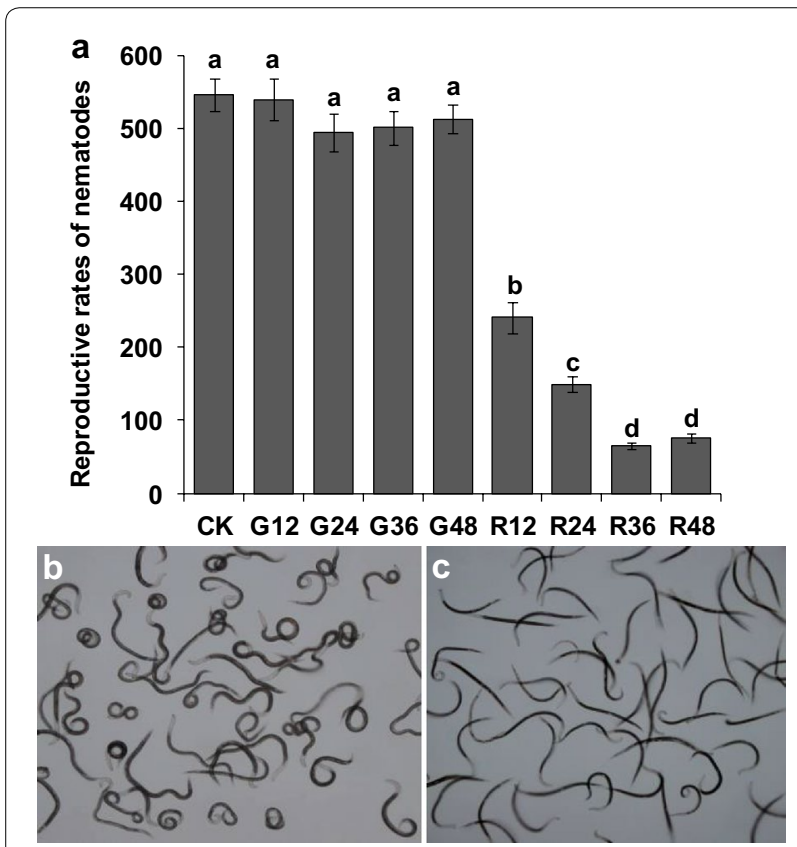

Fig. 8 Phenotypes and reproduction of Radopholus similis after RNAi. a Reproductive rates of $R$. similis extracted from carrot disks at 50 days after inoculation with 30 females. CK: untreated nematodes; G12-G48: nematodes treated with non-endogenous egfp dsRNA for 12 h, 24 h, $36 \mathrm{~h}$ and $48 \mathrm{~h}$, respectively; R12-R48: nematodes treated with Rs-cps dsRNA for 12 h, 24 h, 36 h and 48 h, respectively. The bars indicate the standard errors of the mean $(n=5)$ and different letters indicate significant differences $(p<0.05)$ between treatments. b, c The phenotypic observation of $R$. similis. Nematodes were soaked in Rs-cps dsRNA (b) and non-endogenous egfp dsRNA solutions (c) for $36 \mathrm{~h}$ plants inoculated with untreated (2224) and egfp dsRNAtreated nematodes (2148). The latter two treatments did not differ significantly $(p>0.05)$ (Fig. 9d). The degree of root damage was much lower in tomato plants inoculated with Rs-cps dsRNA-treated nematodes than in the control plants inoculated with untreated and egfp dsRNAtreated nematodes, but spots and local rot caused by nematodes treated with Rs-cps dsRNA were observed on these plant roots. The root systems of the tomato plants inoculated with untreated and e $g f p$ dsRNA-treated nematodes were significantly smaller and severely damaged compared to those of healthy plants, indicating obvious root rot (Fig. 9e). The inoculation tests thus demonstrated that the pathogenicity of $R$. similis was significantly decreased after treatment with Rs-cps dsRNA for $36 \mathrm{~h}$, whereas the pathogenicity of $R$. similis was not impacted by treatment with non-specific e $g f p$ dsRNA.

\section{Discussion}

Cysteine proteinases play important biological roles in nematodes and many other animal parasites [9-12, 17]. In vitro RNAi-induced gene silencing has been successfully applied to free-living nematode C.elegans and the migratory endoparasitic plant-parasitic nematode $B$. xylophilus, without the addition of a neurostimulant [31, 32]. Delivery of dsRNA via ingestion is difficult in sedentary endoparasites such as cyst nematodes and rootknot nematodes because these second-stage juveniles (J2s) feed only following the establishment of a feeding site inside the root, and do not ingest substances prior to this stage [27]. However, uptake of dsRNA soaking solution has been induced in these nematode J2s by adding neurostimulant $[27,28,44]$. RNAi has been applied to $R$. similis by soaking the nematodes in dsRNA solution that does not contain neurostimulant [19, 20,34]. The ability of the neurostimulant octopamine to enhance the ingestion of soaking solution by $R$. similis and the potential detrimental effects of octopamine on nematode activity have not been evaluated. Tan et al. [33] reported that the percentage of activity Pratylenchus thornei was reduced by only $12 \%$ after the worms were incubated with $100 \mathrm{mM}$ octopamine for $16 \mathrm{~h}$ compared to the control group. Tan et al. also demonstrated that more than $90 \%$ of both $P$. thornei and $P$. zeae were dead after only $4 \mathrm{~h}$ of incubation in $1 \%$ resorcinol (another neurostimulant) [33]. In this study, using FITC as a marker, we demonstrated that $R$. similis can ingest dsRNA soaking solution without stimulation by a neurostimulant, similar to $C$. elegans and $B$. xylophilus [27, 31,32]. We also confirmed that the neurostimulant octopamine did not significantly enhance the ingestion of dsRNA soaking solution by $R$. similis but instead had a detrimental effect on nematode activity. In addition, the dsRNA soaking solution may diffuse into the 

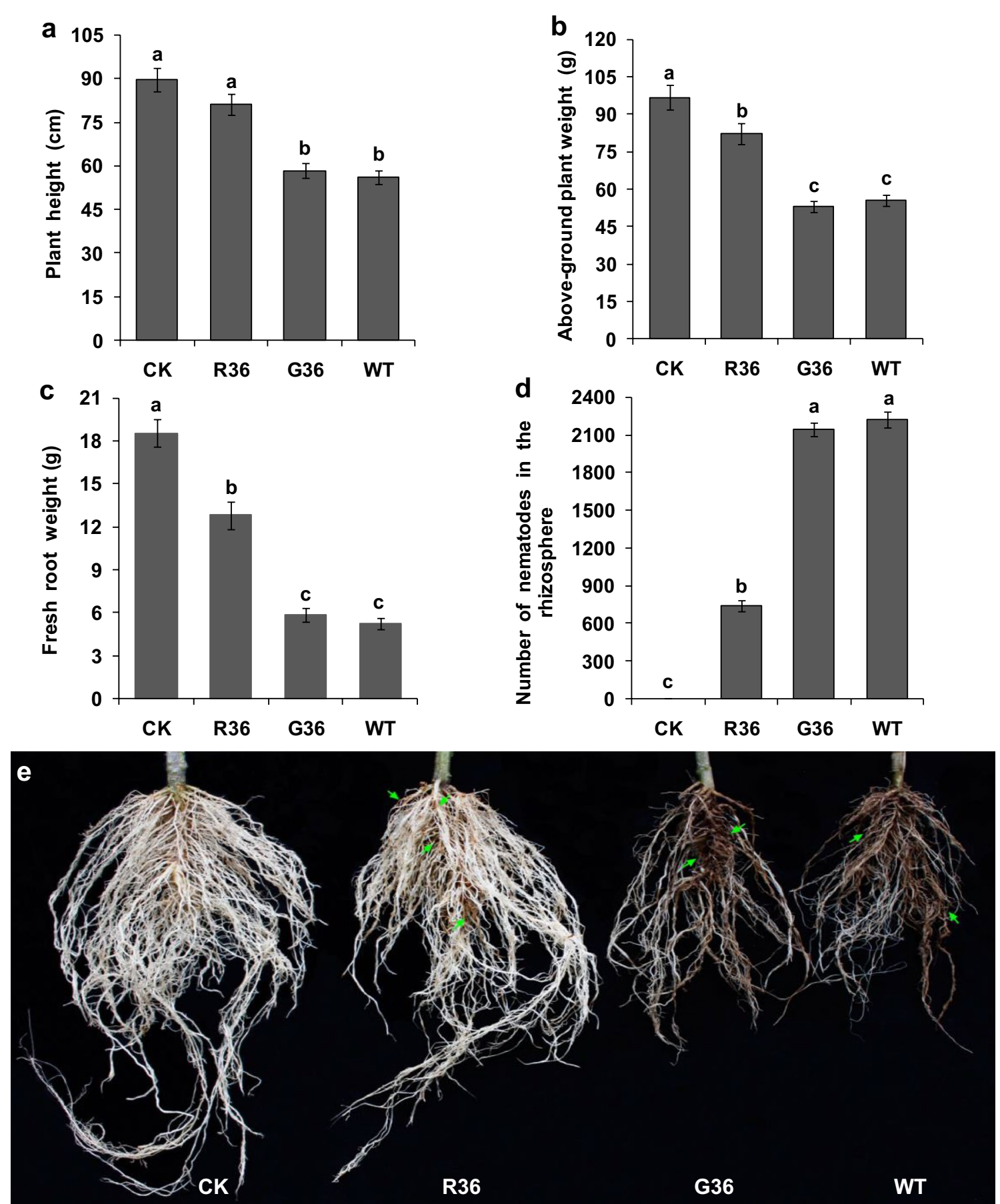

Fig. 9 Effect of RNAi on the pathogenicity of Radopholus similis. Plant height (a), above-ground plant weight (b), fresh root weight (c), number of nematodes in the rhizosphere (d) and root infection symptoms (e) of tomato plants 2 months after inoculation with 1000 nematodes treated under different conditions. CK: uninoculated healthy tomato plants; R36: inoculated with nematodes treated with Rs-cps dsRNA for 36 h; G36: inoculated with nematodes treated with non-endogenous egfp dsRNA for 36 h; WT: inoculated with untreated nematodes. The bars indicate the standard errors of the mean $(n=5)$, and different letters indicate significant differences $(p<0.05)$ between treatments

body of $R$. similis not only via the esophageal lumen but also via the amphids, excretory duct, vagina, anus, cloacal orifice and egg shells.
The $c b$ gene is mainly expressed in the intestines in C. elegans [45], A. cantonensis [46] and Haemonchus contortus [47] and in the cecal epithelial cells, digestive 
tract and reproductive system in F. gigantic [11]. Li et al. [20] reported that $R s-c b-1$ is expressed in the esophageal glands, intestines and gonads of females, the testes of males, and juveniles and eggs in $R$. similis. Hashmi et al. [10] reported that $\mathrm{Ce}-\mathrm{cl}-1$ is widely expressed in the intestines, hypodermal cells and eggshells of C. elegans. Guiliano et al. [14] confirmed that $c l$ is highly expressed in the esophageal glands of $B$. malayi and $B$. pahang $i$ infective third-stage larvae. In the plant parasitic nematode $M$. incognita, $\mathrm{Mi}$-cl-1 is expressed in the intestines of young and mature female nematodes [30]. In this study, the expression and localization of Rs-cps in R. similis were associated with the biological functions of cathepsin. The expression of Rs-cps in the esophageal glands of $R$. similis may facilitate the disruption of plant defensive responses, the establishment of a parasitic relationship, and the rapid digestion of host cells to obtain the nutrients necessary for metabolism and other physiological functions. These findings are also consistent with the functions of esophageal secretions that have been documented in other plant parasitic nematodes. The secretions of esophageal glands produced by plant parasitic nematodes are thought to play key roles throughout the process of parasitism $[48,49]$. Rs-cps was located in the eggs and reproductive system of $R$. similis, possibly because CPS plays important roles in development, reproduction and cell differentiation in $R$. similis. The females of $R$. similis are responsible for both infection and reproduction; Rs-cps expression is therefore highest in females. Thakur et al. [21] reported that the expression level of $\mathrm{Ha}$-cps was also highest in females of $H$. avenae. Rs-cps expression was significantly higher in infective juveniles than in eggs, likely because the successful destruction of host defense responses and the establishment of a parasitic relationship are a precondition for other functions to be implemented by $R$. similis. In this study, Rs-cps expression was lowest in eggs, $42.5 \%$ of the expression level in females. This result reveals that CPS may also play important roles in embryo formation and cell differentiation in $R$. similis. Previous studies have shown that $R s-c b-1$ plays vital roles in reproduction, development and pathogenesis in $R$. similis $[19,20]$. B-, L- and S-like cathepsin belong to the cysteine protease family, and they share a close genetic relationship and similar structures. Therefore, these proteases may have similar biological functions during processes such as infection and pathogenesis in nematodes.

To further define the functions of Rs-cps and to explore the possibility of using this promising target for controlling $R$. similis, an RNAi experiment was performed in this study. Treatment with Rs-cps dsRNA significantly decreased the expression levels of Rs-cps and the reproductive rates of $R$. similis compared to the control groups. The silencing efficiency of Rs-cps was highest and the reproductive rate of $R$. similis was lowest on carrot disks after treatment with Rs-cps dsRNA for $36 \mathrm{~h}$. In the subsequent pot experiments, the pathogenicity of $R$. similis to tomato plants was also significantly reduced after treatment with Rs-cps dsRNA for $36 \mathrm{~h}$. These results are consistent with the tissue localization of Rs-cps in $R$. similis. Therefore, we confirmed that Rs-cps plays key roles in reproduction and pathogenesis in $R$. similis and that CPS might be a promising target for controlling this nematode. Here we first report the use of RNAi in studying the functions of $c p s$ in plant parasitic nematodes and suggests a promising new target for controlling $R$. similis. An RNAi effect could be generated in nematodes by feeding on transgenic plants expressing a specific target gene dsRNA [20, 27, 38]. Therefore, these findings support applications aimed at controlling plant parasitic nematodes by constructing $R s$-cps plant RNAi vectors and obtaining transgenic plants that express specific hairpin dsRNAs of reproduction-, parasitism- and pathogenesisrelated genes and merit further investigation.

\section{Conclusions}

This is the first work to examine the functions of $c p s$ from $R$. similis. Rs-cps mRNA was expressed in the esophageal glands and ovaries of females, the esophageal glands, testes and vas deferens of males and the eggs of $R$. similis. $R s$-cps was expressed at varying levels in all developmental stages of $R$. similis. The expression level of Rs-cps was significantly suppressed in nematodes and that the reproductive capability and pathogenicity of $R$. similis were significantly reduced after RNAi. These results indicated that Rs-cps plays important roles in the reproduction, parasitism and pathogenesis of $R$. similis and could be used as a promising target for controlling plant parasitic nematodes.

\section{Authors' contributions}

$\mathrm{KW}, \mathrm{YL}$ and $\mathrm{HX}$ conceived and designed the experiments; $\mathrm{KW}, \mathrm{YL}$ and DWW performed the experiments; KW, YL, XH, CLX and HX analyzed the data; KW, $Y L$ and $H X$ wrote the manuscript. All authors read and approved the final manuscript

\section{Author details \\ ${ }^{1}$ Laboratory of Plant Nematology and Research Center of Nematodes of Plant Quarantine, Department of Plant Pathology, South China Agricultural Uni- versity, Guangzhou 510642, China. ${ }^{2}$ Department of Plant Pathology, Henan Agricultural University, Zhengzhou 450002, China.}

\section{Acknowledgements}

This work was funded by National Natural Science Foundation of China (No. 31071665 and No. 31371920).

\section{Competing interests}

The authors declare that they have no competing interests.

Received: 25 February 2016 Accepted: 2 June 2016

Published online: 10 June 2016 


\section{References}

1. Cotton J, Van Riel H. Quarantine, problems and solutions. In: Evans K, Trudgill DL, Webster JM, editors. Plant parasitic nematodes in temperate agriculture. Wallingford: CAB International; 1993. p. 593-607.

2. Smith IM, Charles LMF. Distribution maps of quarantine pests for Europe Wallingford: CABI Publisher; 1998.

3. O'Bannon JH. Worldwide dissemination of Radopholus similis and its importance in crop production. J Nematol. 1977;9:16-25.

4. Richardson PN, Grewal PS. Nematode pests of glasshouse crops and mushrooms. In: Evans K, Trudgill DL, Webster JM, editors. Plant parasitic nematodes in temperate agriculture. Wallingford: CABI International; 1993. p. 515-6.

5. Luc M, Sikora RA, Bridge J. Plant parasitic nematodes in subtropical and tropical agriculture. Wallingford: CABI Publisher; 2005.

6. Gowen SR. Some considerations of problems associated with the nematode pests of bananas. Nematropica. 1979;9:79-91.

7. Urwin PE, Lilley CJ, McPherson MJ, Atkinson HJ. Characterization of two cDNAs encoding cysteine proteinases from the soybean cyst nematode Heterodera glycines. Parasitology. 1997;114:605-13.

8. Britton C, Murray L. A cathepsin L protease essential for Caenorhabditis elegans embryogenesis is functionally conserved in parasitic nematodes. Mol Biochem Parasitol. 2002;122:21-33.

9. Carmona C, Dowd AJ, Smith AM, Dalton JP. Cathepsin L proteinase secreted by Fasciola hepatica in vitro prevents antibody-mediated eosinophil attachment to newly excysted juveniles. Mol Biochem Parasitol. 1993:62:9-17.

10. Hashmi S, Britton C, Liu J, Guiliano DB, Oksov Y, Lustigman S. Cathepsin $L$ is essential for embryogenesis and development of Caenorhabditis elegans. J Biol Chem. 2002:277:3477-86.

11. Meemon K, Grams R, Vichasri-Grams S, Hofmann A, Korge G, Viyanant V, et al. Molecular cloning and analysis of stage and tissue-specific expression of cathepsin B encoding genes from Fasciola gigantica. Mol Biochem Parasitol. 2004;136:1-10.

12. Malagón D, Díaz-López M, Benítez R, Adroher FJ. Cathepsin B- and L-like cysteine protease activities during the in vitro development of Hysterothylacium aduncum (Nematoda: Anisakidae), a worldwide fish parasite. Parasitol Int. 2010;59:89-92.

13. Britton C, Murray L. Cathepsin L protease (CPL-1) is essential for yolk processing during embryogenesis in Caenorhabditis elegans. J Cell Sci. 2004:117:5133-43.

14. Guiliano DB, Hong X, McKerrow JH, Blaxter ML, Oksov Y, Liu J, et al. A gene family of cathepsin L-like proteases of filarial nematodes are associated with larval molting and cuticle and eggshell remodeling. Mol Biochem Parasitol. 2004;136:227-42.

15. Lustigman S, McKerrow JH, Shah K, Lui J, Huima T, Hough M, et al. Cloning of a cysteine protease required for the molting of Onchocerca volvulus third stage larvae. J Biol Chem. 1996;271:30181-9.

16. Law RH, Smooker PM, Irving JA, Piedrafita D, Ponting R, Kennedy NJ, et al. Cloning and expression of the major secreted Cathepsin B-Like protein from juvenile Fasciola hepatica and analysis of immunogenicity following liver fluke infection. Infect Immun. 2003;71:6921-32.

17. Han YP, Li ZY, Li BC, Sun X, Zhu CC, Ling XT, et al. Molecular cloning and characterization of a cathepsin B from Angiostrongylus cantonensis. Parasitol Res. 2011;109:369-78.

18. Li DL, Li Y, Xie H, Xu CL, Huang X. Cloning and analysis of cathepsin B gene of Radopholus similis. J Zhejiang Univ. 2013;39:26-33.

19. Li Y, Xie H, Xu CL, Li DL, Zhang C. RNAi effect of Cathepsin B gene on reproduction of Radopholus similis. Sci Agric Sin. 2010;43:1608-16.

20. Li Y, Wang K, Xie H, Wang DW, Xu CL, Huang X, et al. Cathepsin B cysteine proteinase is essential for the development and pathogenesis of the plant parasitic nematode Radopholus similis. Int J Biol Sci. 2015:11:1073-87.

21. Thakur PK, Kumar M, Kumar J, Gantasala NP, Rao U. Structural and functional analysis of cathepsin S of Heterodera spp: a promising candidate for its control. Indian J Exp Biol. 2014;52:223-31.

22. Fire A, Xu S, Montgomery MK, Kostas SA, Driver SE, Mello CC. Potent and specific genetic interference by double-stranded RNA in Caenorhabditis elegans. Nature. 1998:391:806-11.
23. Guo S, Kemphues KJ. Par-1, a gene required for establishing polarity in $C$. elegans embryos, encodes a putative Ser/Thr kinase that is asymmetrically distributed. Cell. 1995;81:611-20.

24. Cogoni C, Macino G. Gene silencing in Neurospora crassa requires a protein homologous to RNA-dependent RNA polymerase. Nature. 1999;399:166-9.

25. Misquitta L, Paterson BM. Targeted disruption of gene function in Drosophila by RNA interference (RNA-i): a role for nautilus in embryonic somatic muscle formation. Proc Natl Acad Sci USA. 1999;96:1451-6.

26. Wianny F, Zernicka-Goetz M. Specific interference with gene function by double-stranded RNA in early mouse development. Nat Cell Biol. 2000;2:70-5.

27. Urwin PE, Lilley CJ, Atkinson HJ. Ingestion of double-stranded RNA by preparasitic juvenile cyst nematodes leads to RNA interference. Mol Plant Microbe Interact. 2002;15:747-52.

28. Rosso MN, Dubrana MP, Cimbolini N, Jaubert S, Abad P. Application of RNA interference to root-knot nematode genes encoding esophageal gland proteins. Mol Plant Microbe Interact. 2005;18:615-20.

29. Chen Q, Rehman S, Smant G, Jones JT. Functional analysis of pathogenicity proteins of the patato cyst nematode Globodera rostochiensis using RNAi. Mol Plant Microbe Interact. 2005;18:621-5.

30. Shingles J, Lilley CJ, Atkinson HJ, Urwin PE. Meloidogyne incognita: molecular and biochemical characterisation of a cathepsin L cysteine proteinase and the effect on parasitism following RNAi. Exp Parasitol. 2007;115:114-20.

31. Li XD, Zhuo K, Luo M, Sun L, Liao J. Molecular cloning and characterization of a calreticulin CDNA from the pinewood nematode Bursaphelenchus xylophilus. Exp Parasitol. 2011;128:121-6.

32. Wang XR, Cheng X, Li YD, Zhang JA, Zhang ZF, Wu HR. Cloning arginine kinase gene and its RNAi in Bursaphelenchus xylophilus causing pine wilt disease. Eur J Plant Pathol. 2012;134:521-32.

33. Tan JACH, Jones MGK, Fosu-Nyarko J. Gene silencing in root lesion nematodes (Pratylenchus spp.) significantly reduces reproduction in a plant host. Exp Parasitol. 2013;133:166-78.

34. Zhang C, Xie H, Xu CL, Cheng X, Li KM, LiY. Differential expression of Rs-eng-1b in two populations of Radopholus similis (Tylenchida: pratylecnchidae) and its relationship to pathogenicity. Eur J Plant Pathol. 2012:133:899-910.

35. Fallas GA, Sarah JL. Effect of storage temperature on the in vitro reproduction of Rahodpholus similis. Nematropica. 1994;24:175-7.

36. Arshad W, Haq IU, Waheed MT, Mysore KS, Mirza B. Agrobacterium-mediated transformation of tomato with rolB gene results in enhancement of fruit quality and foliar resistance against fungal pathogens. PLoS One. 2014:9:e96979.

37. Saitou N, Nei M. The neighbor-joining method: a new method for reconstructing phylogenetic trees. Mol Biol Evol. 1987:4:406-25.

38. Li Y, Wang K, Xie H, et al. A nematode calreticulin, Rs-CRT, is a key effector in reproduction and pathogenicity of Radopholus similis. PLoS One. 2015;10:e0129351.

39. De Boer JM, Yan Y, Smant G, Davis EL, Baum TJ. In-situ hybridization to messenger RNA in Heterodera glycines. J Nematol. 1998;30:309-12.

40. Cheng X, Xiang Y, Xie H, Xu CL, Xie TF, Zhang C, et al. Molecular Characterization and functions of fatty acid and retinoid binding protein gene (Ab-far-1) in Aphelenchoides besseyi. PLoS One. 2013;8:e66011.

41. Jacob J, Vanholme B, Haegeman A, et al. Four transthyretin-like genes of the migratory plant-parasitic nematode Radopholus similis: members of an extensive nematode-specific family. Gene. 2007:402:9-19.

42. Hannon GJ. RNAi: a guide to gene silencing. New York: Cold Spring Harbor Laboratory Press; 2003.

43. Kaplan DT, Vanderspool MC, Opperman CH. Sequence tag site and host range assays demonstrate that Radopholus similis and $R$. citrophilus are not reproductively isolated. J Nematol. 1997:29:421-9.

44. Bakhetia M, Charlton W, Atkinson HJ, McPherson MJ. RNA interference of dual oxidase in the plant nematode Meloidogyne incognita. Mol Plant Microbe Interact. 2005;18:1099-106.

45. Ray C, Mckerrow JH. Gut-specific and developmental expression of a Caenorhabditis elegans cysteine protease gene. Mol Biochem Parasitol. 1992;51:239-49. 
46. Cheng M, Yang X, Li Z, He H, Qu Z, He A, et al. Cloning and characterization of a novel cathepsin B-like cysteine proteinase from Angiostrongylus cantonensis. Parasitol Res. 2012;110:2413-22.

47. Pratt D, Armes LG, Hageman R, Reynolds V, Boisvenue RJ, Cox GN. Cloning and sequence comparisons of four distinct cysteine proteases expressed by Haemonchus contortus adult worms. Mol Biochem Parasitol. 1992;51:209-18.
48. Hussey RS, Davis EL, Baum TJ. Secrets in secretions: genes that control nematode parasitism of plants. Braz J Plant Physiol. 2002;14:183-94.

49. Vanholme B, De Meutter J, Tytgat T, Van Montagu M, Coomans A, Gheysen G. Secretions of plant-parasitic nematodes: a molecular update. Gene. 2004;332:13-27.

\section{Submit your next manuscript to BioMed Central and we will help you at every step:}

- We accept pre-submission inquiries

- Our selector tool helps you to find the most relevant journal

- We provide round the clock customer support

- Convenient online submission

- Thorough peer review

- Inclusion in PubMed and all major indexing services

- Maximum visibility for your research

Submit your manuscript at

www.biomedcentral.com/submit 